\title{
Performance of the Ring Imaging Cherenkov Detector of DELPHI
}

\author{
W. Adam et al \\ Presented by G. Lenzen
}

\begin{abstract}
The general purpose particle detector DELPHI at the Large Electron Positron collider at CERN was built to give the complete information of each event. DELPHI uses ring imaging Cherenkov counters to provide hadron identification in most of the momentum range below $40 \mathrm{GeV} / c$ and over almost the full solid angle. Charged particles traversing gaseous and liquid fluorocarbon radiators create photons used for Cherenkov angle reconstruction. Some of the design features of the detector will be presented, with emphasis on the experience which was gained in the operation of these large systems. The hadron identification power of the ring imaging Cherenkov detector closely meets the main design values. Data processing and performance of the detector will be discussed using dimuon events collected during 1994. Pion rejection factors for kaon tagging will be shown.
\end{abstract}

Contribution to the $7^{\text {th }}$ International Wire Chamber Conference, Vienna, Austria, February 13-17, 1995 


\section{PERFORMANCE OF THE RING IMAGING CHERENKOV DETECTOR OF DELPHI}

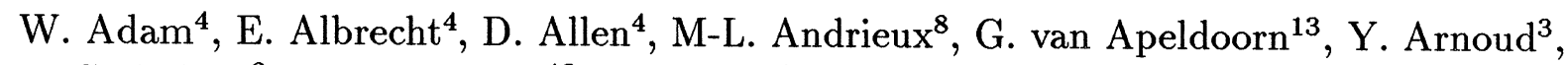
C. Aubret ${ }^{2}$, A. Augustinus ${ }^{13}$, P. Baillon ${ }^{4}$, M. Battaglia ${ }^{16}$, D. Bloch ${ }^{5}$, O. Botner ${ }^{17}$, C. Bourdarios ${ }^{4}$, J. M. Brunet ${ }^{2}$, A. Buys ${ }^{4}$, P. Carrié ${ }^{4}$, P. Cavalli ${ }^{8}$, G. Cerutti ${ }^{4}$, M. Chevry ${ }^{11}$, E. Christophel ${ }^{5}$, E. Dahl-Jensen ${ }^{12}$, G. Damgaard ${ }^{12}$, N. Dimitriou ${ }^{6}$, B. D’Almagne ${ }^{9}$, M. Davenport ${ }^{4}$, F. Delbecque ${ }^{4}$, J. Dolbeau $^{2}$, M. Dracos ${ }^{5}$, M. Dris ${ }^{14}$, T. Ekelöf ${ }^{17}$, J. P. Engel ${ }^{5}$, D. Fassouliotis ${ }^{14}$, T. A. Filippas ${ }^{14}$, E. Fokitis ${ }^{14}$, F. Fontanelli ${ }^{7}$, A. Fontenille ${ }^{8}$, D. Fraissard ${ }^{4}$, H. Fürstenau ${ }^{4}$, J. Garcia ${ }^{15}$, E. N. Gazis ${ }^{14}$, D. Gillespie ${ }^{4}$, V. Gracco ${ }^{7}$, L. Guglielmi ${ }^{2}$, F. Hahn ${ }^{18}$, S. Haider ${ }^{13}$, A. Hallgren ${ }^{17}$, W. Hao ${ }^{13}$, K. Huet ${ }^{11}$, P. Ioannou ${ }^{1}$, S. Ilie ${ }^{4}$, S. Jääskeläinen ${ }^{4}$, C. Joram ${ }^{4}$, P. Juillot ${ }^{5}$, E. Karvelas ${ }^{6}$, S. Katsanevas ${ }^{1}$, E. Katsoufis ${ }^{14}$, N. Kjaer ${ }^{12}$, P. M. Kluit ${ }^{13}$, B. Koene ${ }^{13}$, C. Kourkoumelis ${ }^{1}$, G. Lecoeur ${ }^{4}$, G. Lenzen ${ }^{18}$, L-E. Lindqvist ${ }^{17}$, A. López Agúera ${ }^{15}$, D. Loukas ${ }^{6}$, S. Maltezos ${ }^{14}$, A. Markou ${ }^{6}$, J. Medbo $^{17}$, J. Michalowski ${ }^{10}$, F. Montano ${ }^{7}$, G. Mourgue ${ }^{4}$, B. S. Nielsen ${ }^{12,4}$, R. Nicolaidou ${ }^{1}$, Th. D. Papadopoulou ${ }^{14}$, A. Petrolini ${ }^{7}$, D. Poutot ${ }^{2}$, G. Polok $^{10}$, H. Rahmani ${ }^{14}$, M. Reale ${ }^{18}$, L. K. Resvanis ${ }^{1}$, G. Sajot ${ }^{8}$, M. Sannino ${ }^{7}$, E. Schyns ${ }^{18}$, S. Squarcia ${ }^{7}$, P. Stassi ${ }^{8}$, R. Strub ${ }^{5}$, J. Thadome ${ }^{18}$, G. E. Theodosiou ${ }^{6 \dagger}$,

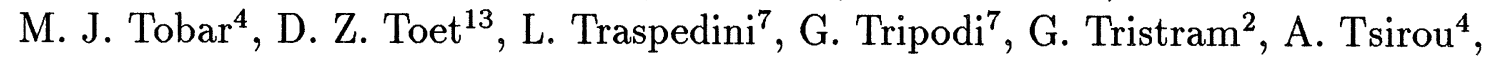
O. Ullaland ${ }^{4}$ and E. Zevgolatakos ${ }^{6}$

Contribution to the 7th International Wire Chamber Conference, Vienna, Austria, February 13-17, 1995

Presented by G.Lenzen

\footnotetext{
1) University of Athens, Physics Department, Physics Laboratory, Solonos Str. 104, GR-10680 Athens, Greece

2) Collège de France, Laboratoire de Physique Corpusculaire, 11 place Marcelin-Berthelot, F-75231 Paris, France

3) CENS Centre d'Etudes Nucléaires de Saclay, DSM/DAPNIA/SPP, Service de Physique des Particules, F-91191 Gifsur-Yvette Cedex, France

4) CERN, CH-1211 Geneva 23, Switzerland

5) Centre de Recherche Nucléaire, IN2P3-CNRS/ULP, BP28, F67037 Strasbourg Cédex2, France

6) Institute of Nuclear Physics, N.C.S.R. 'Demokritos', P.O. Box 60228, GR-15310 Aghia Paraskevi, Attiki, Greece

7) Dipartimento di Fisica, Università di Genova and INFN, Via Dodecaneso 33, I-16146 Genova, Italy

8) Institut des Sciences Nucléaires, Université de Grenoble 1, F-38026 Grenoble, France

9) LAL Laboratoire de l'Accélérateur Linéaire, Université de Paris-Sud (Paris XI), Bâtiment 200, F-91405 Orsay Cédex, France

10) High Energy Physics Laboratory, Inst. of Nucl. Physics, Ul. Kawiory 26a, PL-30055 Krakow 30, Poland

11) Université de Mons-Hainaut, Service de Physique des Particules Elémentaires, Faculté des Sciences, Av. Maistriau 15, B-7000 Mons, Belgium

12) Niels Bohr Institute, Blegdamsvej 17, DK-2100 Copenhagen, Denmark

13) NIKHEF-H, Postbus 41882, NL-1009 DB Amsterdam, The Netherlands

14) NTU National Technical University, Physics Laboaratory II, 9 Heroes of Polytechneion Street, Zografou, GR-15780 Athens, Greece

15) Facultad de Ciencias, Universidad de Santander, av. de los Castros, E-39005 Santander, Spain

16) Research Institute for High Energy Physics, SEFT, Siltavuorenpenger 20c, SF-00170 Helsinki, Finland

17) Dept. of Radiation Sciences, University of Uppsala, P.O. Box 535, S-751 21 .Uppsala, Sweden

18) Fachbereich Physik, University of Wuppertal, Gauss-Str. 20, D-42097 Wuppertal, Germany t Deceased
} 
DELPHI, a DEtector with Lepton, Photon and Hadron Identification [1], at the Large Electron-Positron (LEP) collider at CERN is a general purpose detector which combines a three dimensional track localization and a good energy resolution with an excellent identification of leptons, photons and hadrons. The layout of the DELPHI detector is shown in fig.1. DELPHI is equipped with two independent Ring Imaging Cherenkov (RICH) detectors for hadron identification, the Barrel [2] and the Forward [3] RICH. The cylindrical Barrel RICH covers the polar angle range $40^{\circ}<\vartheta<140^{\circ}$, while the Forward RICH consists of two endcaps and covers the polar angle ranges $15^{\circ}<\vartheta<35^{\circ}$ and $145^{\circ}<\vartheta<165^{\circ}$. The large momentum range up to $45 \mathrm{GeV} / c$ of particles produced in $Z^{0}$ decays necessitates two Cherenkov radiator systems with complementary refractive indices. Both Barrel and Forward RICH counters use the same design principles, although their geometrical layouts are different. The Barrel RICH is fully operational since 1991, and the Forward RICH, a staged item at LEP start-up, is fully installed and operational since 1993.

When traversing liquid and gaseous radiators, a charged relativistic particle creates Cherenkov photons. The emission angle $\theta$ of the Cherenkov radiation relative to the track of the particle is given by $\cos \theta=1 /(\beta n(\omega))$, where $\beta=v / c$ is the velocity of the particle relative to the light speed in vacuum and $n(\omega)$ is the refractive index of the medium at frequency $\omega$. Therefore, if $n(\omega)$ is known for a given medium, the measurement of $\theta$ gives the velocity of the particle. The number of photons emitted is proportional to $\sin ^{2} \theta$. The identity or mass $m$ of this particle can however only be determined by simultaneous measurements of its Cherenkov emission angle $\theta$ and its momentum $p$. Applying the above equation the mass can be calculated : $m=p \sqrt{n^{2}(\omega) \cos ^{2} \theta-1}$.

Ultraviolet Cherenkov light created in the two media is detected with a common photon detector system consisting of segmented time projection chambers. It has two basic parts: a drift box with UV-grade quartz windows and an electron detector. Mirrors are used in the gas radiator to focus the Cherenkov photons back onto the photon detector, while the light produced in the liquid radiator falls directly onto the photon detection plane. The UV photons are converted into free electrons by the photosensitive agent TMAE, Tetrakis-diMethylAmino-Ethylene $\mathrm{C}_{2}\left[\left(\mathrm{CH}_{3}\right)_{2} \mathrm{~N}\right]_{4}{ }^{1)}$, added to the drift gas of the photon detector. The photoelectrons drift in a constant electric field towards the end region of the box, where they are detected by a multiwire proportional chamber (MWPC). The measured drift time and the hit addresses of anode wires and cathode strips allow a three dimensional reconstruction of the conversion point of each individual Cherenkov photon with an accuracy better than $1 \mathrm{~mm}$.

Non-flammable and non-toxic fluorocarbons [4] were chosen as Cherenkov radiators because of their good UV transparency in the photon detection range limited by the TMAE photo-ionization threshold of $5.64 \mathrm{eV}$ and the transmission cut-off of the quartz windows of the photon detector at $7.50 \mathrm{eV}$. They give rise to only small chromatic aberrations, which is an important feature, since the photon detectors register photons indiscriminately over a quite large energy range.

The RICH counters are sandwiched between tracking devices which determine the impact point of the particle on the RICH detector and its momentum in the $1.2 \mathrm{~T}$ magnetic field of DELPHI. The Barrel RICH is situated between the main tracking device, the Time Projection Chamber, and the Outer Detector, while the Forward RICH is located

1) TMAE : RSA Corp., Ardsley, NY 10502, USA 
between two large MWPC systems, the Chambers A and B (see fig.1). Both tracking systems deliver a combined momentum resolution of $\mathrm{dp} / \mathrm{p}<8 \%$ for most tracks, which is sufficient for particle identification with the RICH counters.

The accuracy $\sigma_{\theta}$ with which the Cherenkov angle $\theta$ is measured determines the particle identification power of the RICH detectors. The refractive indices of the Cherenkov radiators depend on temperature and pressure variations. Moreover, they may change due to different compositions of the fluids caused by leaks and diffusion. Therefore efficient monitoring, control and calibration systems are implemented in the RICH counters ensuring a stable, safe and long-term operation of the different subsystems. All control and monitoring information of the relevant detector parameters is written onto a database and combined with the physics data during the data processing.

The development of the DELPHI RICH detector system was initiated by the work reported in $[5,6]$, while later developments are summarized in $[7,8,9,10]$.

\section{Design features and general operation}

The Barrel RICH has a cylindrical detector volume $(2.46 \mathrm{~m}$ inner diameter, $3.94 \mathrm{~m}$ outer diameter, length $3.5 \mathrm{~m}$ ) divided into left and right sides by a central support wall. It includes a $24 \mathrm{~m}^{3}$ gas radiator volume filled with saturated perfluoropentane $\mathrm{C}_{5} \mathrm{~F}_{12}{ }^{2}$ ). A total of 48 liquid radiator trays of $1 \mathrm{~cm}$ thickness are each filled with 51 of saturated perfluorohexane $\mathrm{C}_{6} \mathrm{~F}_{14}{ }^{3)}$. The refractive index $n$ at $7 \mathrm{eV}$ and $10^{5} \mathrm{~Pa}$ is 1.00172 for $\mathrm{C}_{5} \mathrm{~F}_{12}$, and 1.283 for $\mathrm{C}_{6} \mathrm{~F}_{14}$. The driftgas of the photon detector is a mixture of $75 \%$ methane $\left(\mathrm{CH}_{4}\right)$ and $25 \%$ ethane $\left(\mathrm{C}_{2} \mathrm{H}_{6}\right)$, with about $0.1 \%$ of the photosensitive agent TMAE. The photon detector consists of 24 pairs of quartz drift boxes with a volume of 501 per pair. The concentration of TMAE is equivalent to its saturated vapour pressure at $28^{\circ} \mathrm{C}$ which results in a short absorption length for Cherenkov photon compared to the thickness of the drift box. A total of 288 parabolical mirrors reflect the Cherenkov photons, created in the gas radiator, back onto the drift boxes. Heating circuits mounted on the walls of the Barrel RICH cylinder maintain the temperature of this part of the detector to about $40^{\circ} \mathrm{C}$ in order to avoid condensation of the perfluoropentane, which has a boiling point of $28^{\circ} \mathrm{C}$.

The Forward RICH in each of the two DELPHI endcaps consists of a gas radiator volume of $4 \mathrm{~m}^{3}$ of saturated perfluorobutane $\mathrm{C}_{4} \mathrm{~F}_{10}{ }^{4)}\left(n=1.00153\right.$ at $\left.7 \mathrm{eV}, 10^{5} \mathrm{~Pa}\right)$, a liquid radiator system of 36 individual $1 \mathrm{~cm}$ thick radiators of about $11 \mathrm{each}$, filled with $\mathrm{C}_{6} \mathrm{~F}_{14}$, and a photon detection system with 12 drift volumes of $201 \mathrm{each}$, filled with pure ethane $\left(\mathrm{C}_{2} \mathrm{H}_{6}\right)$ and an amount of TMAE, corresponding to a vapour pressure of $24^{\circ} \mathrm{C}$. Sixty spherically shaped mirrors image the Cherenkov photons from the gas radiator onto the drift boxes. Each Forward RICH endcap is divided in azimuth into 12 modular sectors. The MWPCs are mounted along the radial direction on the border of each $30^{\circ}$ sector. The Forward $\mathrm{RICH}$ containers do not require heating circuits because $\mathrm{C}_{4} \mathrm{~F}_{10}$ has a low boiling point of $-1.7^{\circ} \mathrm{C}$.

\subsection{Photon detection}

All three RICH detectors are situated in the 1.2 T magnetic field of the DELPHI experiment. In the Barrel RICH the magnetic field is parallel to the drift field $\left(E_{d}=0.35 \mathrm{kV} / \mathrm{cm}\right)$ of the photon detector systems, while in the Forward RICH the drift

2) $\mathrm{C}_{5} \mathrm{~F}_{12}: 3 \mathrm{M}$ Co. St.Paul, MN 55144, USA, under the trademark FC87 or PF5050

3) $\mathrm{C}_{6} \mathrm{~F}_{14}: 3 \mathrm{M}$ Co., under the trademark FC72 or PF5060

4) $\mathrm{C}_{4} \mathrm{~F}_{10}: 3 \mathrm{M}$ Co., under the trademark CEA410 
field $\left(E_{d}=1 \mathrm{kV} / \mathrm{cm}\right.$ ) is perpendicular to the magnetic field, leading to a Lorentz angle of about $50^{\circ}$ between the electron drift direction and the electrical drift field. The homogeneous drift field is produced by means of conductive strips on both the inside and the outside of the $4 \mathrm{~mm}$ thick quartz windows of the drift boxes. These strips, $0.1 \mathrm{~mm}$ wide with a pitch of $3 \mathrm{~mm}$, are produced by vacuum deposition of chromium and copper. An additional field cage around the drift boxes reinforces the field homogeneity inside the box. The tapering of the drift volume gap $(4.2 \mathrm{~cm}$ to $6.2 \mathrm{~cm})$ increases the photoelectron collection efficiency by containing the transverse diffusion of electrons produced near the quartz windows of the drift box.

In order to detect single photoelectrons with more than $90 \%$ efficiency the MWPCs have are operated at a high gas amplification of $2 * 10^{5}$. UV blinds optically shield neighbouring wires from secondary photons propagating from the avalanches at the anode wire. The photoelectrons have a maximal drift length of $150 \mathrm{~cm}$ in the Barrel and $50 \mathrm{~cm}$ in the Forward RICH. The chambers provide three dimensional reconstruction of photon conversion points from anode and cathode signal addresses and from the drift time.

\subsection{Radiators and fluid systems}

The refractive indices of the gaseous and liquid fluorocarbons are matched in such a way that there is practically no gap in the momentum coverage. Although the refractive index of $\mathrm{C}_{4} \mathrm{~F}_{10}$ is low in comparison to that of $\mathrm{C}_{5} \mathrm{~F}_{12}$, its better UV transparency and the longer radiator length in the Forward RICH $(58 \mathrm{~cm})$ in comparison to the Barrel RICH $(38 \mathrm{~cm})$ lead to about the same number of photoelectrons for the two RICH detectors.

The pressure inside the $\mathrm{C}_{5} \mathrm{~F}_{12}$ gas radiator of the Barrel $\mathrm{RICH}$ is set to a fixed value of $1030 \pm 0.5 \mathrm{hPa}$ with $\mathrm{n}=1.00192$ at $7 \mathrm{eV}$. Therefore the Barrel RICH needs a pressure regulation system for both the drift gas and the liquid radiator circuits in order to avoid rupture of the quartz plates of the drift boxes and the liquid radiator trays. The Barrel $\mathrm{RICH}$ liquid radiator system pressure is set to $-45 \mathrm{hPa}$ with respect to its gas radiator reference, while the drift gas system runs at about $7 \mathrm{hPa}$ above this reference pressure. All subsystems of the Forward RICH are referenced to the atmospheric pressure by bubblers.

The fluid systems handling the drift gas, liquid, and gas radiator circuits of both Barrel and Forward RICH counters are completely independent of each other. Because of the high cost of the fluorocarbon liquids, the gas and liquid radiator circuits of all RICH counters recirculate the fluids after removal of oxygen and water admixture. Their drift gases are non-recirculating circuits and the gas is vented to the atmosphere after TMAE condensation. All subsystems of the RICH counters are purged with inert gases before operation. An extensive description of the fluid systems can be found in [11].

\section{3}

\section{The monitoring, control and calibration systems}

Working in the UV region imposes many constraints on the materials and special construction techniques have been developed. After some optimization the RICH detector was successfully designed, constructed and brought to full operation. Numerous tests on different materials were performed before they were used in the assembly of the detector, though for instance the influence of the perfluorocarbon liquids on the composites and plastic materials used in the RICH detectors became apparent only after several years of operation. All quartz plates needed for the construction of the liquid radiator trays and drift boxes have a flatness of about $0.1 \mu \mathrm{m}$ and their UV transparency was tested by a monochromator system to be higher than $60 \%$ at $170 \mathrm{~nm}$. The requirement on the reflectivity of all mirrors was higher than $80 \%$ between $160 \mathrm{~nm}$ and $230 \mathrm{~nm}$. 
The monitoring, control and calibration systems of the RICH counters keep track of the RICH behaviour during LEP operation. A stable operation of the different subsystems and monitoring of the relevant detector parameters is crucial in order to achieve the necessary good data quality. For reliable hadron identification it is necessary to know many detector parameters because of their influence on the Cherenkov angle measurement. Therefore the correct operating temperatures and pressures, the gas parameters at delivery, the high and low voltages for the MWPCs, and the very high voltages for the drift fields, are under computerized control. A fast feedback to the operator is realized by having the control and monitoring devices interlinked via a computer network. Parameters are automatically logged to the general DELPHI database to be used in the offline analysis. Online data checking facilities monitor the electronics readout chain. In addition to these continuously controlled and monitored parameters, the UV transparencies of the Cherenkov gases and liquids and the photon conversion depths in TMAE are measured with monochromator systems [4] typically once a week. The sound velocities in the radiator gases are surveyed by sonar-based devices [14] which provide a rough monitoring of the composition and stability of the $\mathrm{N}_{2}-\mathrm{C}_{5} \mathrm{~F}_{12}$ or $\mathrm{N}_{2}-\mathrm{C}_{4} \mathrm{~F}_{10}$ gas mixture. In the future, the refractive indices of the radiator gases in the far UV region will be directly measured by a Fabry-Pérot type refractometer [15].

The RICH counters in DELPHI are equipped with calibration systems [12, 13] which use photons to ionize the TMAE inside the drift boxes. In the Barrel RICH a pulsed UV lamp is situated outside the detector. The light is distributed via quartz optical fibres onto the face of the drift boxes at very precisely defined spots. In the case of the Forward RICH a deuterium lamp for each drift box is placed inside the gas vessels and the light distributed to equally well defined spots on the quartz windows by quartz fibres. The Barrel RICH calibration system is operating at a rate of $0.3 \mathrm{~Hz}$ during data taking. The Forward RICH calibration is performed between physics runs. The calibration data are used to determine the drift velocities with systematic accuracies better than $0.05 \%$ for the Barrel and about $0.2 \%$ for the Forward RICH systems. The continuous operation of the Barrel RICH calibration system also allows monitoring of changes in the electron attenuation length, which has been measured to be around 10 meters. Any distortions in the drift field are measured and monitored as well. In addition, the Lorentz angle in the Forward RICH is calculated from the calibration data.

\section{Operational experience}

Since the beginning of 1993 all RICH detectors of the DELPHI experiment are operational, although the Barrel RICH liquid radiator system had to be switched off in the last part of 1993 due to leaks. These were to a large extent cured before the 1994 run.

It has turned out that the long-term contact between the perfluorocarbon liquids and certain composite and plastic materials used inside the RICH detectors [16] leads to dimensional changes and modification of mechanical properties. The leaks that developed in 1993 between the liquid and gas radiators was found to be due to a loss of elasticity of PVC washers on pressostat units at the Barrel RICH endplate.

At the end of 1993 one Barrel RICH double drift box and one double liquid radiator tray were dismounted from the detector and the shrunk wire frame and the voltage degrader bar were replaced. Small leaks were found inside the liquid radiator units where some of the Rilsan pipes for filling and emptying the radiators were found to be completely disconnected from the radiator tray again due to shrinkage. These leaks cannot be cured without dismantling all liquid radiator trays of the Barrel $\mathrm{RICH}$. The operation of the 
detector is however not jeopardized by this. Some leaks were also detected in a part of the Forward RICH liquid radiator systems leading to a migration of $\mathrm{C}_{6} \mathrm{~F}_{14}$ into the $\mathrm{C}_{4} \mathrm{~F}_{10}$ gas radiator.

Corona discharges observed in a Barrel RICH drift box may have been caused by detensioned field shaping wires touching each other due to shrinkage of the mounting frame. For the 1994 data taking period a reduction of the Barrel RICH drift voltage from $80 \mathrm{kV}$ to $54 \mathrm{kV}$ made further corona discharges unlikely. The increased diffusion of the photoelectrons drifting in the lower electrical field of $0.35 \mathrm{kV} / \mathrm{cm}$ does not appear to affect the Cherenkov angle resolution significantly. Therefore for the time being, a repair of further wire frames is not foreseen.

The leaks between the Barrel RICH liquid and gas radiator systems $\left(0.8 \mathrm{l} /\right.$ day $_{6} \mathrm{~F}_{14}$ into the 3501 liquid $\mathrm{C}_{5} \mathrm{~F}_{12}$ gas radiator system, $1.2 \mathrm{l}$ /day liquid $\mathrm{C}_{5} \mathrm{~F}_{12}$ into the $400 \mathrm{l}$ $\mathrm{C}_{6} \mathrm{~F}_{14}$ liquid radiator system) lead to mixtures of the two perfluorocarbons of varying composition which result in changes of the refractive indices and of the condensation points. In 1994 an online separation plant for $\mathrm{C}_{5} \mathrm{~F}_{12}-\mathrm{C}_{6} \mathrm{~F}_{14}$ liquid mixtures was built using the method of fractional distillation [18]. The $\mathrm{C}_{6} \mathrm{~F}_{14}$ contamination in the $\mathrm{C}_{5} \mathrm{~F}_{12}$ liquid can be removed to a large extent through repeated emptying of the evaporator of the gas radiator system. For the Forward RICH detector the $\mathrm{C}_{6} \mathrm{~F}_{14}$ is separated from the $\mathrm{C}_{4} \mathrm{~F}_{10}$ gas by a two-stage distillation device. These precautions have stabilized the level of fluid mixtures to a level where their operation is safe and the effect on the performance is small.

The analysis of ionizing tracks traversing the photon detectors can reveal distortions in the drift field. The RICH track position is measured with a typical accuracy of $2 \mathrm{~mm}$ and can be compared with tracks extrapolated from the central tracking detectors of DELPHI. The results are sensitive to any disturbances occuring in either the photon detectors or the central tracking. Hence, it is possible to monitor the relative alignment with high precision. It has been observed that a few photon detectors show anomalies in the difference between the two measurements. These anomalies extend over a few millimeters, and are possibly due to small electrical short circuits between some adjacent potential strips of the Barrel RICH field degrader. The anomalies cause an error on the drift coordinate which is on average below $0.5 \mathrm{~mm}$. For some photon detectors the effects are larger and therefore corrected for. Even for these detectors the magnetic field ensures, however, that drift field imperfections cause only a loss of $2 \%$ in the number of detected photoelectrons [17].

During the 1994 run all RICH systems were successfully operating without interuption. A data sample of about $1.5 * 10^{6}$ hadronic $Z^{0}$ events was recorded. The quality of these data taken with the RICH detectors will be demonstrated in the next chapter.

\section{5}

Data processing and performance

The particle identification power of the RICH detector is directly linked to the accuracy in the reconstruction of the Cherenkov photons. The performance of this complex device is to a large extent governed by the accurate determination of all parameters which influence the photon emission-conversion and the detection of the photoelectrons. Optimal performance also requires a high precision in the alignment of the RICH detector subsystems relative to the DELPHI tracking devices. Selected high momentum $Z^{0} \rightarrow \mu^{+} \mu^{-}$ events are used to align the DELPHI detectors. A software alignment package [19] for the RICH systems minimizes the difference between measured and expected Cherenkov angles for each individual photoelectron created by these dimuon events. This is done by slightly varying the positions of the detector elements (such as radiators, photon detectors 
and mirrors) or the trajectory of the track. The UV-light calibration systems of the Forward and Barrel RICH counters provide a detailed understanding of the drift properties of the photon detectors. After the minimization procedure the optimal performance of the whole analysis chain is verified by reprocessing the muon sample with the corrected database. In addition different algorithms are applied on the photoelectron level in order to improve the signal to background ratio $[20,21]$. The rejection power for background photoelectrons is more than $60 \%$ for both the gas and the liquid radiators, at the cost of a signal loss of only about $10 \%$.

The importance of the alignment procedure is demonstrated in fig. 2 which shows the clear improvement in resolution of Cherenkov angles for the Forward RICH gas radiator system. Fig. 3 shows the Barrel RICH Cherenkov angle resolution plotted against the mirror number for dimuon events in the 1994 data obtained with the gas radiator.

The obtained resolutions are summarized in table 1 . The numbers are in good agreement with those expected from detailed simulation programs. The observed behaviour in the resolution on the gas Cherenkov angle measurement follows very closely the expected $1 / \sqrt{N_{p e}}$ law, while in the resolution on the liquid radiator angle measurement a small systematic error of about $4 \mathrm{mrad}$ is still observed.

\begin{tabular}{|c|c|c|c|c|c|c|}
\hline & \multicolumn{3}{|c|}{ Liquid Radiator } & \multicolumn{3}{c|}{ Gas Radiator } \\
\cline { 2 - 7 } & $\begin{array}{c}\text { Photo- } \\
\text { electrons }\end{array}$ & $\sigma(\mathrm{mrad})$ & $\begin{array}{c}\text { Ch-Angle } \\
\sigma(\mathrm{mrad})\end{array}$ & $\begin{array}{c}\text { Photo- } \\
\text { electrons }\end{array}$ & $\sigma(\mathrm{mrad})$ & $\begin{array}{c}\text { Ch-Angle } \\
\sigma(\mathrm{mrad})\end{array}$ \\
\hline Barrel RICH & 12.0 & 13.6 & 5.2 & 8.4 & 4.5 & 1.9 \\
\hline Forward RICH & 6.7 & 11.4 & 6.1 & 7.2 & 2.6 & 1.5 \\
\hline
\end{tabular}

Table 1: Preliminary results on number of photoelectrons detected, single photoelectron resolution and Cherenkov angle resolution for $45 \mathrm{GeV} / \mathrm{c}$ muons in the 1994 data sample.

The refractive indices of the Cherenkov radiators are obtained through fits performed on the data, using $Z^{0} \rightarrow \mu^{+} \mu^{-}$decays. Moreover, variations in the refractive index can be directly deduced from the known contamination of $\mathrm{C}_{5} \mathrm{~F}_{12}$ in the $\mathrm{C}_{6} \mathrm{~F}_{14}$ liquid. In fig. 4 the variations of the Cherenkov angle for the Barrel RICH liquid radiator are shown over the running period 1994.

After having aligned the RICH detectors with a selected set of dimuon events the expected Cherenkov signal for each track of known momentum from $Z^{0}$ decays can be computed by the reconstruction program for all mass hypotheses, namely $\mathrm{e}, \mu, \pi, \mathrm{K}$ and p. The signal is characterized by the value of the Cherenkov angle, its uncertainty and the number of photoelectrons. The so-called ring identification mode of particle identification is based on the comparison between the measured and expected Cherenkov signal for each mass hypothesis. Charged particles with a velocity below the Cherenkov threshold $\beta<1 / n$ emit no Cherenkov light. In the veto identification mode kaons and protons can be separated from lighter particles in the momentum range where kaons and protons are below the threshold while the lighter particles already emit photons.

Fixed parameters of the detector hardware which enter into the calculation of the expected RICH signal, such as the optical properties of the quartz windows or mirrors, and characteristics of the fluids, like UV transparencies or electron absorption length, are passed to the offline analysis through the DELPHI data-base.

Due to the variety of topologies encountered in the different physics channels, the simulation is the only tool which permits a detailed evaluation of the identification per- 
formances of the detectors. In the full simulation of the RICH all detector constants are taken into account. The predictions are extensively cross-checked with the detector response observed in real data using special samples of particles of known mass coming from resonance decays. For momenta higher than $5 \mathrm{GeV} / \mathrm{c}$, the particle identification provided by the RICH counters is also cross-checked with the measurement of the specific ionization loss $(\mathrm{dE} / \mathrm{dx})$ in the DELPHI Time Projection Chamber. Depending on the requirements of the physics channel of interest, the tagging performance can be tuned in order to enhance either its efficiency or its purity [22].

Fig. 5 presents as an example for the identification power of the RICH counter the results obtained for kaon tagging over a large momentum range after the liquid ring, the gas veto, and the gas ring modes have been combined. The tagging efficiency is defined as the fraction of particles that were assigned the correct tag in a sample selected by specific quality cuts. The contamination is defined as the fraction of particles which are attributed the wrong tag and the rejection power is defined as the ratio of these two numbers. The triangles represent the kaon efficiency, while the ovals give the contamination of protons tagged as kaons. The associated pion rejection factors are 30 in the liquid, and 10 in the gas ring mode. For momenta around $8 \mathrm{GeV} / \mathrm{c}$, the identification relies on the gas veto mode only. In this zone, both kaons and protons are below the Cherenkov threshold and cannot be discriminated. The pion rejection factor in this restricted momentum region is 15.

\section{Conclusions}

The Forward and Barrel RICH detectors in the DELPHI experiment are fully operational with both liquid and gaseous radiators. The stability of all RICH subsystems during data taking is maintained by computerized control and monitoring features, which are interlinked via a computer network which also updates the database. Disturbances in the operation of the RICH counters were mainly caused by the perfluorocarbons which induce dimensional and mechanical modifications on the composites and plastic materials. Leaks between the gas and the liquid radiators result in changes of the refractive indices and of the condensation points. The operation of the RICH detectors was however successfully adapted to these circumstances by lowering the drift field voltage and by the addition of distillation devices in the fluid circuits. Systematic effects due to these disturbances could be observed but the amount of recorded data makes detailed studies and efficient offline corrections possible. A good resolution in the reconstruction of the Cherenkov angle could be achieved after offline alignment of the detector components. The analysis techniques developed for the DELPHI RICH detector have been applied to the data taken during the 1992 and 1993 LEP runs. A detailed analysis of the large 1994 data sample is under way. The excellent quality of the data closely meets the values the detector was designed for. The DELPHI RICH detectors are now used as a standard hadron identification tool, in particular for heavy flavour physics analyses.

\section{$7 \quad$ Acknowledgements}

The contribution and dedicated work of many people, -from the development to the final realization of the DELPHI RICH detectors-, is gratefully acknowledged. We wish to thank all our collaborators for their support and help during so many years. We are especially indebted to the technical collaborators of the participating institutes for their excellent work. We are grateful to our funding agencies for their continuous support of the project. 


\section{References}

[1] P.Aarnio et al. (DELPHI Collaboration), Nucl.Instr. and Meth. A 303 (1991) 233-276

[2] G. van Apeldoorn et al., IEEE Trans.Nucl.Sci, Vol.41, No.4, 866-870, August 1994

[3] W.Adam et al., IEEE Trans.Nucl.Sci, Vol.41, No.4, 856-861, August 1994

[4] G.Lenzen et al., Nucl.Instr. and Meth. A 343 (1994) 268-272

[5] J.Séguinot, T.Ypsilantis, Nucl.Instr. and Meth. 142 (1977) 377-391

[6] W.Dulinski et al., Nucl.Instr. and Meth. A 252 (1986). 418-422 and references therein

[7] P.Beltran et al., Proc.Int.Symp. on Position Detectors in High-Energy Physics, Dubna, 1987, JINR D1-13-88-172, p.286

[8] R.Arnold et al., Nucl.Instr. and Meth. A 270 (1988) 255-288 and references therein

[9] R.Arnold et al., Nucl.Instr. and Meth. A 270 (1988) 289-319

[10] E.G.Anassontzis et al., Nucl.Instr. and Meth. A 323 (1992) 351-362 and references therein

[11] W.Adam et al., Nucl.Instr. and Meth. A 343 (1994) 68-73

[12] P.Adrianos et al., Nucl.Instr. and Meth. A 294 (1990) 424-430

[13] A.Markou et al., Internal report, DELPHI 91-95, RICH-45, (1991)

[14] G.Hallewell et al., Nucl.Instr. and Meth. A 264 (1988) 219

[15] E.Fokitis et al., The Fabry-Pérot interferometer for the DELPHI ring imaging Cherenkov detector, Como Conference 1994, to be published

[16] E.Albrecht et al., Perfluorocarbon effects on composite and polymeric materials used within RICH detectors, Internal report, DELPHI note, to be published

[17] W.Adam et al., Internal report, DELPHI 94-109, PHYS-426, (1994)

[18] S.Ilie, G.Lenzen, Perfluorocarbon liquids for Cherenkov detectors: separation and purification, Internal report, DELPHI note, to be published

[19] M.Berggren, Internal report, DELPHI 89-81, PROG-146, (1989)

[20] M.Dracos, Thesis, CRN/HE 87-10, Strasbourg, 1987

[21] J.García Perez, A.López Agüera, Nucl.Instr. and Meth. A 343 (1994) 276-278

[22] W.Adam et al., Internal report, DELPHI 94-112, PHYS 429, (1994) 


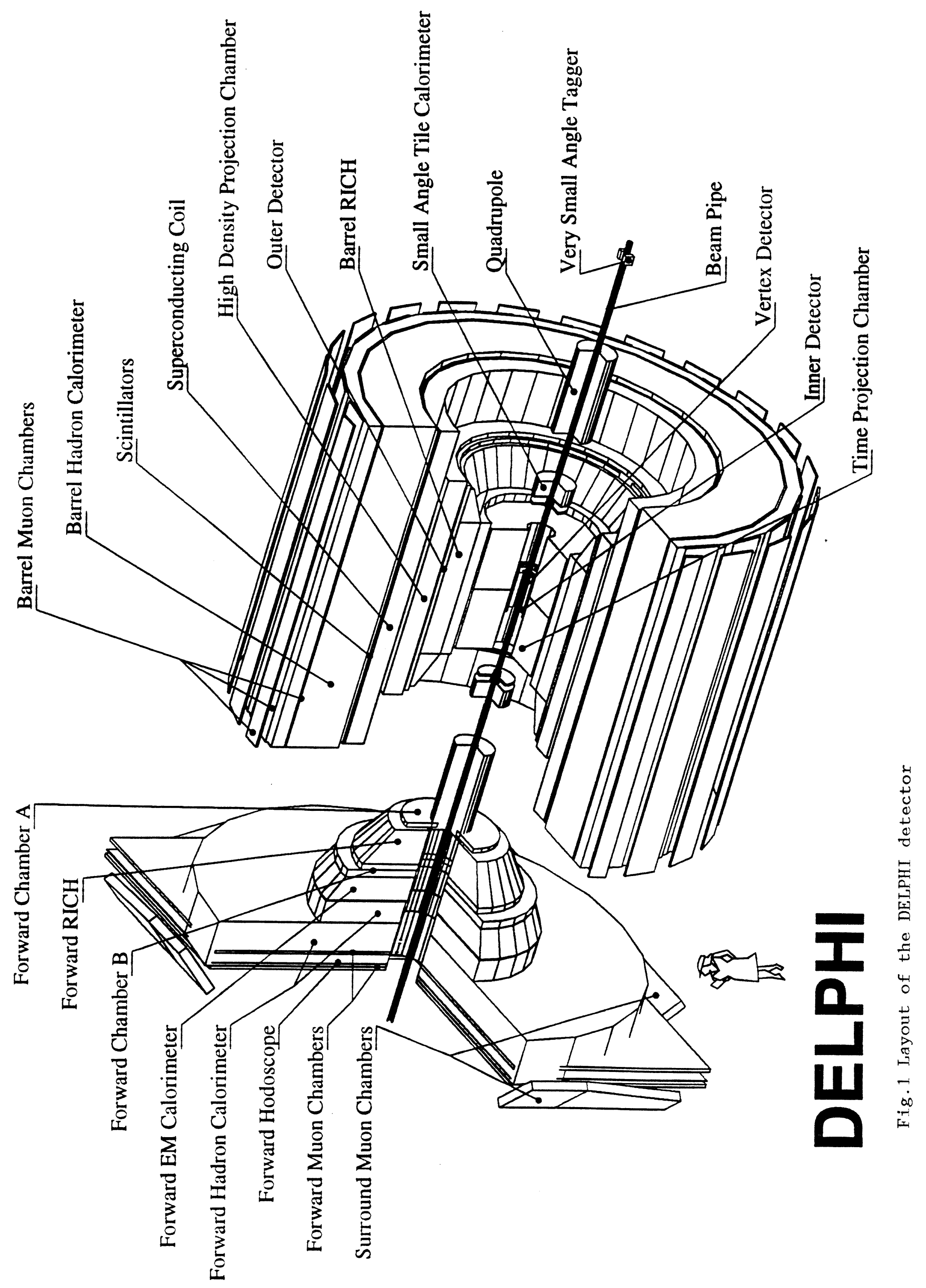




\section{Forward RICH Gas Radiator}

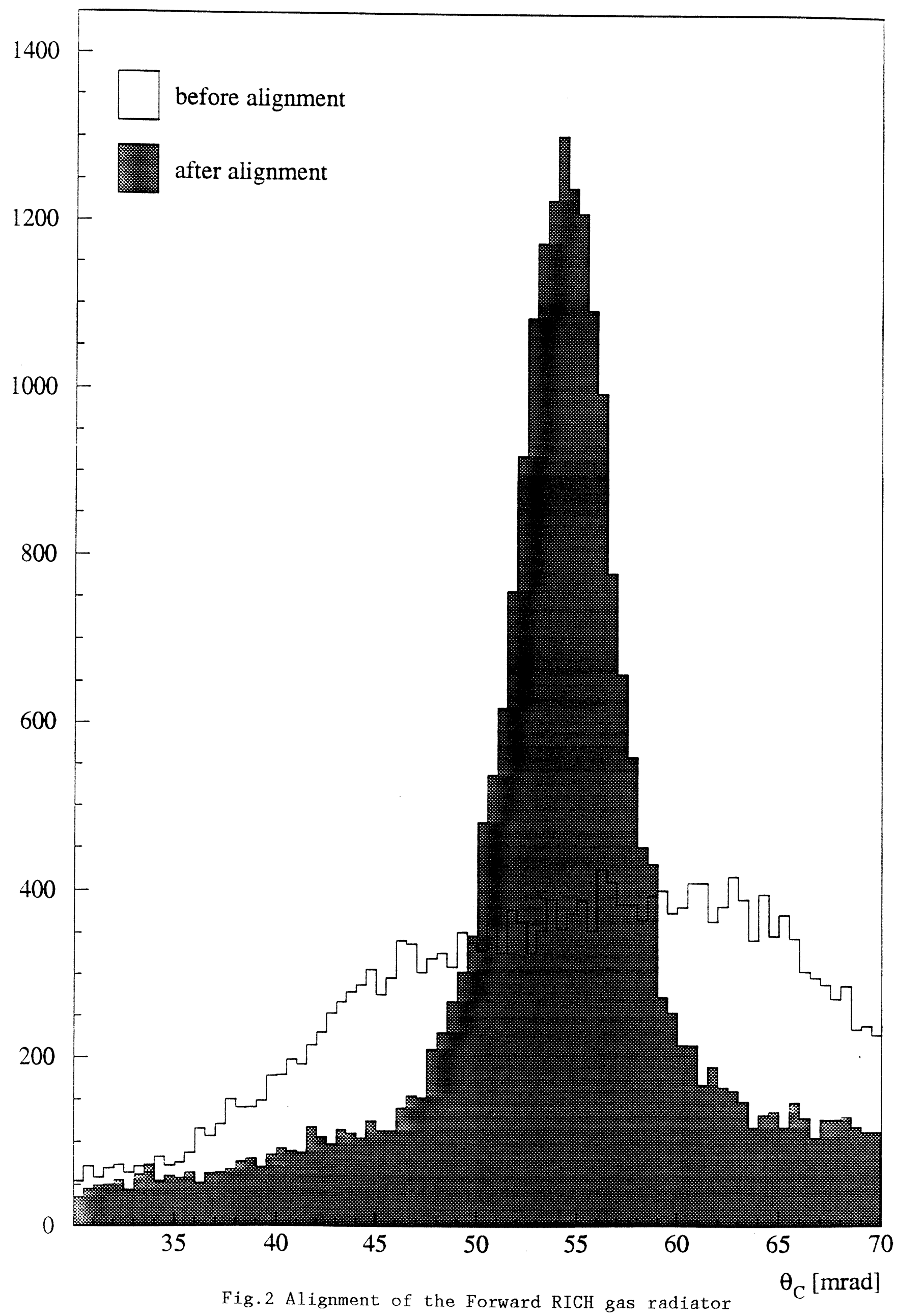




\section{B BRICH ALIGNMENT}

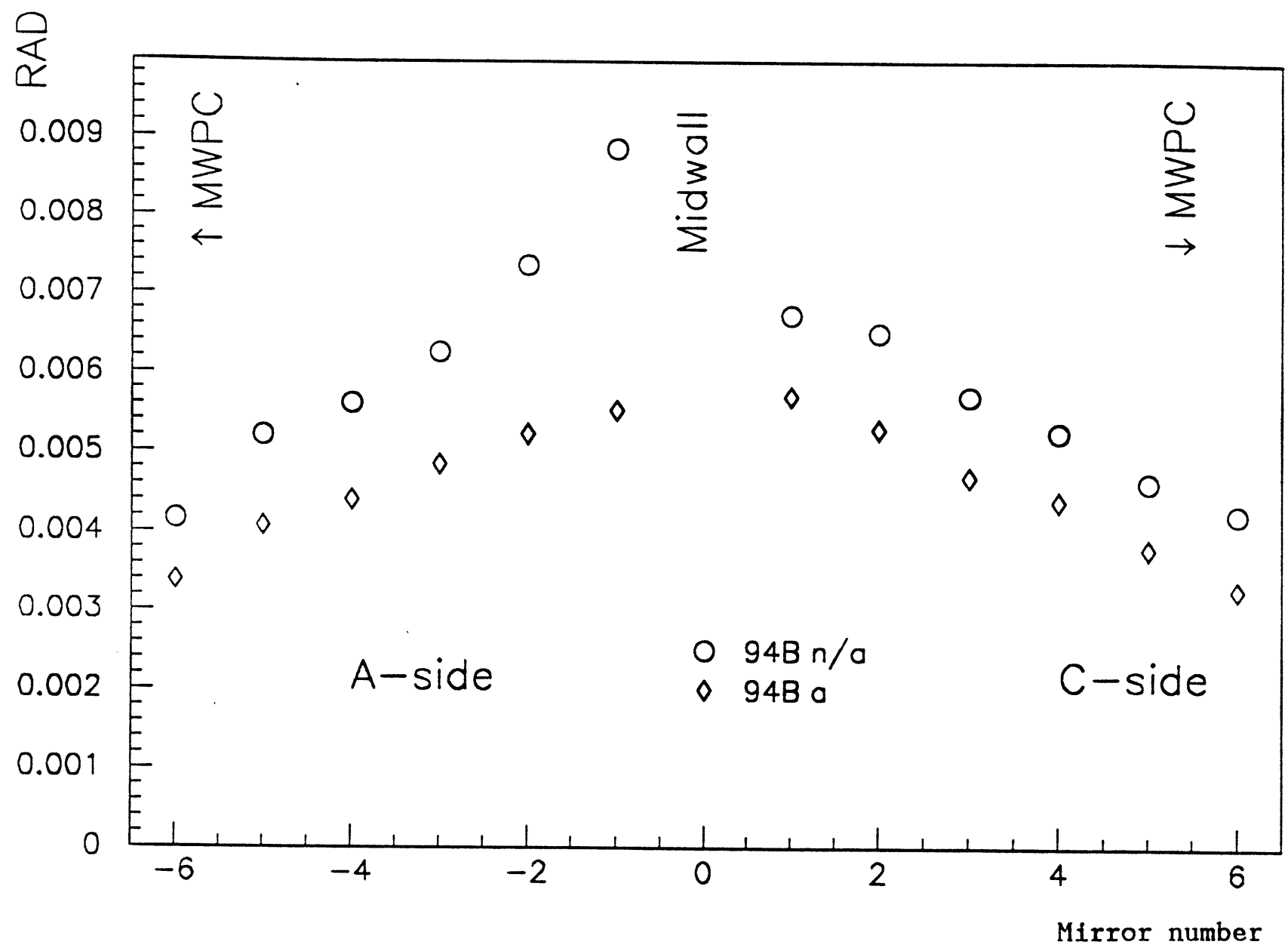

Fig. 3 Cherenkov angle resolution of the Barrel RICH gas radiator

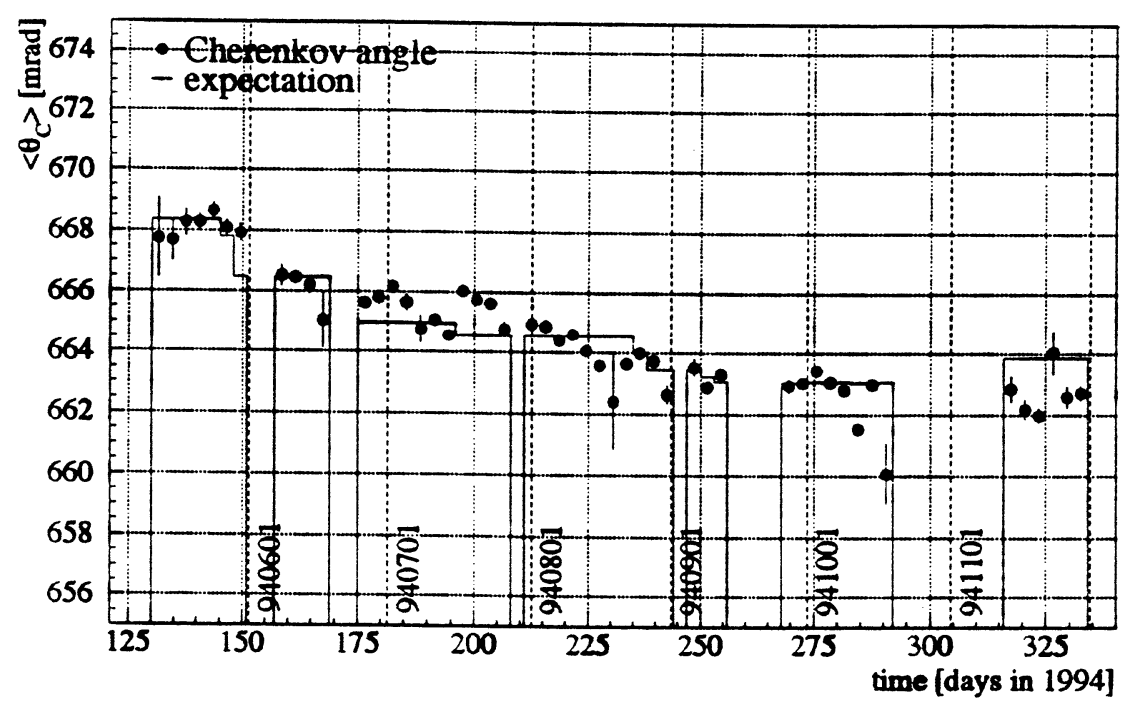

Fig. 4 Time dependence of the Cherenkov angle of the Barre1 RICH 1iquid radiator 


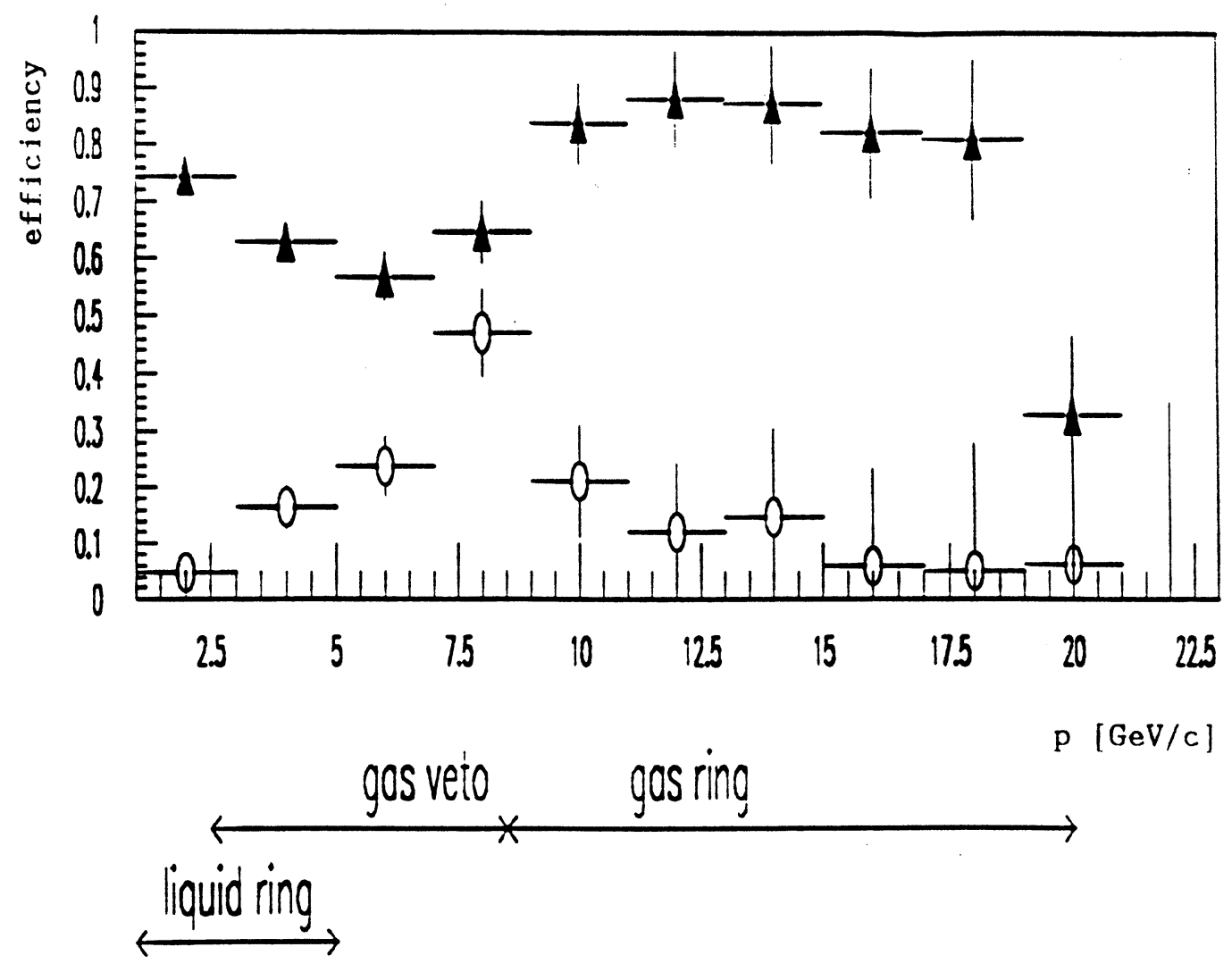

Fig.5 Fractions of kaons (triangles) and protons (ovals) tagged as kaons 